\title{
Frecuencia de enfermedades infecciosas en caninos en la Clínica Veterinaria Docente Cayetano Heredia en el periodo 2014-2017
}

Frequency of infectious diseases in canines at Clínica Veterinaria Docente Cayetano Heredia in the period 2014-2017

\author{
Erika Zuñiga ${ }^{1}$, Clarisa Hinostroza ${ }^{1}$, Renato Zúñiga², Daphne León ${ }^{1}$ \\ RESUMEN
}

El estudio tuvo como objetivo determinar la frecuencia de enfermedades infecciosas confirmadas mediante análisis de laboratorio, en la Clínica Veterinaria Docente Cayetano Heredia $(\mathrm{CVDCH})$ en el periodo 2014 al 2017. Las historias clínicas fueron elegidas bajo los criterios que contengan información legible, completa y diagnóstico confirmatorio de laboratorio de alguna enfermedad infecciosa. Las variables consideradas en el estudio se agruparon en información del paciente (procedencia, grupo etario, sexo, raza y estacionalidad de la atención) y el diagnóstico definitivo de enfermedad(es) infecciosa(s) según su etiología. Se obtuvieron 307 historias clínicas de pacientes con diagnóstico(s) confirmatorio(s) para el periodo de estudio. Los casos fueron más frecuentes en los años 2014 y 2015 y procedían de distritos de Lima Norte. Los pacientes en su mayoría fueron machos adultos de raza definida. En otoño se reportó la mayor frecuencia de casos de enfermedades infecciosas. Los diagnósticos confirmados más frecuentes fueron erhlichiosis $(45,7 \%)$, leptospirosis $(11,5 \%)$, anaplasmosis $(10,6 \%)$, giardiasis $(8,7 \%)$, coccididiosis $(7,8 \%)$, distemper $(7,8 \%)$ y parvovirosis (7,8\%) Así mismo, se halló 11,5\% de presentación de canes diagnosticados con dos o más agentes, siendo la principal infección mixta causada por Erlichia sp. y Anaplasma sp. El estudio provee información acerca de las enfermedades más frecuentes atendidas en la CVDCH siendo una aproximación del comportamiento espacial y temporal de las enfermedades en el área de influencia de la CVDCH.

PALABRAS CLAVE: frecuencia, enfermedades infecciosas, etiología, caninos

Facultad de Medicina Veterinaria y Zootecnia, Universidad Peruana Cayetano Heredia. Lima, Perú.

Laboratorio de Análisis Clínico Vet Support. Lima, Perú. 


\section{SUMMARY}

The objective of the study was to determine the frequency of infectious diseases confirmed by laboratory analysis at the Clínica Veterinaria Docente Cayetano Heredia (CVDCH) in the period 2014 to 2017 . The medical records were chosen considering that they are legible, with complete information and diagnosis laboratory confirmation of infectious disease (s). The variables considered in the study were grouped into patient information (origin, age group, sex, breed, and seasonality of care) and the definitive diagnosis of infectious disease (s) according to their etiology. 307 medical records were obtained from patients with confirmatory diagnosis (es) for the study period. The cases were more frequent in 2014 and 2015 and came from districts of North Lima. Most patients were racedefined adult males. In autumn the highest frequency of cases of infectious diseases was reported. The most frequent diseases were erhlichiosis(45.7\%), leptospirosis (11.5\%), anaplasmosis (10.6\%), giardiasis $(8.7 \%)$, coccididiosis $(7.8 \%)$, distemper $(7.8 \%)$ and parvovirus $(7.8 \%)$. The presentation of dogs diagnosed with two or more agents is $11.5 \%$, being the main mixed infection caused by Erlichia sp. and Anaplasma sp. The study provides information about the most frequent diseases treated in the $\mathrm{CVDCH}$, approximating the spatial and temporal behavior of the diseases in the area of influence of the same.

KEY WORDS: frequency, infectious diseases, etiology, canines

\section{INTRODUCCIÓN}

Los síndromes clínicos inducidos por agentes infecciosos son comunes en la clínica de animales pequeños y son diagnosticado de manera presuntiva a través de la información obtenida de una reseña, anamnesis y examen físico del animal. Sin embargo, el diagnóstico definitivo debe de ser obtenido mediante el uso de pruebas de laboratorio específicas, para así abordar los aspectos terapéuticos, profilácticos y de control zoonótico, si lo hubiera, de forma correcta (Couto, 2005).

Una enfermedad infecciosa puede clasificarse de acuerdo con el agente patógeno causal o etiología infecciosa en bacteriana, viral, fúngica y parasitaria (Greene, 2000). Estos agentes patógenos son capaces de infectar al organismo del paciente y producir una alteración celular, produciendo lesiones de tejidos y órganos que determinan los signos clínicos en el organismo de un hospedador (Schudel y van Gelderen, 2002; Kumar et al., 2010).
Entre las enfermedades infecciosas virales se destacan el Parvovirus y Distemper canino. El síndrome producido por el virus de Parvovirus canino (CPV) destaca por ocasionar trastornos gastrointestinales. Esta enfermedad ha alcanzado frecuencias elevadas en Sudamérica, en Colombia se reporta frecuencias de 70.4\% (Duque et al., 2017) y en Chile de 78\% (Acosta et al., 2015); mientras que en Tarapoto-Perú se reportó $53,3 \%$ de infecciones por CPV en canes con gastroenteritis hemorrágica (Mendoza, 2017). Entre los agentes etiológicos que producen cuadros respiratorios, destaca el Virus del Distemper Canino (CDV). Su frecuencia ha alcanzado niveles de $78 \%$ en albergues del Brasil (Monteiro et al., 2016) y en Lima - Perú se encontró 32,7\% de CDV en caninos no vacunados con signos compatibles con distemper (Soto, 2017).

Entre las enfermedades bacterianas, la leptospirosis destaca por su distribución cosmopolita y potencialmente zoonótica (Silva y Riedemann, 2007). En Colombia se ha demostrado un $41,1 \%$ de seropositividad 
(Rodríguez et al., 2004); $81 \%$ en México (Luna et al., 2008); 14,8\% en Chile (Silva y Riedemann, 2007) y 58\% en Lima - Perú (Siuce et al., 2015).

La ehrlichiosis canina es una de las enfermedades bacterianas más importantes trasmitidas por vectores. Es causada por la bacteria Erhlichia canis y se transmite mediante la picadura de la garrapata Rhipicephalus sanguineus, y se considera una enfermedad infecciosa inmunodepresora de distribución mundial en caninos. En México, se han obtenido un $74,3 \%$ de resultados positivos (Sosa et al., 2013) y de $59,4 \%$ en un estudio retrospectivo en un laboratorio en Lima - Perú (Cusicanqui, 2018).

La anaplasmosis canina es causada por las bacterias Anaplasma phagocytophilum y A. platys es otra de enfermedades transmitidas por garrapatas cuya prevalencia varía geográficamente. En Costa Rica se reporta 6,3\% de positividad a $A$. phagocytophilum (Dolz et al., 2013); mientras que en Colombia alcanza una frecuencia de 33\% (McCown et al., 2015). En Perú se reporta 55.1\% de canes infectados con Anaplasma spp. en el departamento de Lambayeque (Delgado y Montoya, 2018) y de $29,2 \%$ a $A$. platys en el departamento de Lima (Tateishi et al., 2015).

Entre las enfermedades parasitarias de importancia y que también producen infecciones gastrointestinales, se encuentra la causada por el protozoo Giardia spp que se distribuye en niveles variables en los canes de los países sudamericanos (Ballweber et al., 2010) y en Perú se encontró una frecuencia de $16,7 \%$ en un estudio en Lima (Sotelo et al., 2013). Otro ejemplo es la coccidiosis, causada por Isospora spp., es una infección común en canes alcanzando niveles de detección en heces de hasta 19.8\% en Bolivia (Llanos et al., 2010), de 6,4\% en Colombia (Caraballo et al., 2007) y en Chile de 9,2\% (López et al., 2006).
La frecuencia de presentación de las enfermedades infecciosas varía según región o zona geográfica. Tener disponible esta información es importante para poder evaluar el comportamiento de las enfermedades transmisibles y de esta manera prever un mejor manejo terapéutico y profiláctico. La Clínica Veterinaria Docente Cayetano Heredia (CVDCH) de la Facultad de Medicina Veterinaria de la Universidad Peruana Cayetano Heredia (FAVEZ-UPCH) se encuentra ubicada en el Norte de Lima Metropolitana recibe pacientes no solo del mismo distrito sino también de zonas geográficas aledañas, cuya casuística infecciosas no ha sido sistematizada.

El estudio tuvo como objetivo determinar la frecuencia de enfermedades infecciosas confirmadas mediante pruebas de laboratorio, en muestras procedentes de pacientes caninos atendidos en la Clínica Veterinaria Docente Cayetano (CVDCH) durante el periodo 2014 2017.

\section{MATERIAL Y MÉTODOS}

El estudio retrospectivo se realizó en la CVDCH de la FAVEZ-UPCH. Se incluyeron en el estudio todas las historias clínicas con información completa, de canes con diagnóstico de laboratorio confirmatorio de enfermedad infecciosa, atendidos en el periodo 2014 - 2017.

Las variables consideradas en el estudio se clasificaron de la siguiente manera:

Año, se clasificaron según la temporalidad en el que se reportó el caso (2014, 2015, 2016, 2017):

- Procedencia: Se clasificó la procedencia de los pacientes en 3 zonas principales según la clasificación de los distritos de Lima Metropolitana: Lima Norte, Lima Centro y otras (que incluyen Lima Sur, Lima Este y otras zonas geográficas como Barranca 
y Huaral) (Instituto Nacional de Estadística e Informática (INEI), 2014).

- Raza: Para la clasificación se consideró las razas de los canes según la Federación Cinológica Internacional (FCI) y fueron conglomerados con el término raza definida. Además, se consideró a los canes mestizos como otro grupo definido (FCI, 2016; Fascetti y Delaney, 2012).

- Grupo etario: Se clasificó en los diferentes estadios de vida del animal: cachorro, adulto y adulto mayor (Bartges et al., 2012).

- Estacionalidad: Se consideró la estacionalidad según lo intervalos cronológicos definidos convencionalmente en otoño, Invierno, primavera y verano.

La información obtenida de las historias clínicas fue transferida a una base de datos en el programa Microsoft Excel $\AA$, considerando una columna para cada variable seleccionado para el estudio. Para resumir la información se utilizó estadística descriptiva, presentando los resultados en frecuencias absolutas $y$ relativas para cada etiología de la enfermedad distribuidas según las variables de clasificación consideradas en el estudio.

\section{RESULTADOS}

De un total de 6006 historias clínicas en el periodo 2014 - 2017, se obtuvieron $307(5,1 \%)$ historias clínicas con un total de 357 diagnósticos definitivos de enfermedades infecciosas, considerando que 41 pacientes (11,5\% de las historias clínicas revisadas) presentaban dos o más diagnósticos definitivos. Los pacientes en su mayoría fueron de raza definida, sexo macho y estrato etario adulto. La cantidad de historias con diagnóstico definitivo disminuyó en el tiempo, encontrándose en mayor número en los años 2014 y 2015. La mayor frecuencia de casos correspondía a la zona de Lima Norte. La estación del año que presento mayor frecuencia de casos de enfermedades infecciosas fue otoño. El detalle de la distribución de estos casos se presenta en la tabla 1.

Las enfermedades diagnosticadas fueron erhlichiosis $(45,7 \%)$, leptospirosis $(11,5 \%)$, anaplasmosis $(10,6 \%)$, giardiasis $(8,7 \%)$, coccididiosis $(7,8 \%)$, distemper $(7,8 \%)$ y parvovirosis $(7,8 \%)$. La distribución de los casos según las variables de estudio (año de ocurrencia, procedencia, raza, sexo, estrato

Tabla 1. Características demográficas de los pacientes caninos con enfermedades infecciosas que fueron atendidos en la Clínica Veterinaria Docente Cayetano Heredia en el periodo 2014-2017. Lima - Perú.

\begin{tabular}{|c|c|c|}
\hline Variable & $\mathbf{n}$ & $\%$ \\
\hline \multicolumn{3}{|l|}{$\operatorname{Raza}(\mathrm{N}=307)$} \\
\hline Definida & 189 & 61,6 \\
\hline Mestizo & 118 & 38,4 \\
\hline \multicolumn{3}{|l|}{$\operatorname{Sexo}(N=307)$} \\
\hline Macho & 192 & 62,5 \\
\hline Hembra & 115 & 37,5 \\
\hline \multicolumn{3}{|c|}{ Estrato etario $(\mathrm{N}=308)^{1}$} \\
\hline Adulto & 133 & 43,2 \\
\hline Cachorro & 102 & 33,1 \\
\hline Adulto Mayor & 73 & 23,7 \\
\hline \multicolumn{3}{|l|}{ Estación $(\mathrm{N}=311)^{*}$} \\
\hline Otoño & 105 & 33,8 \\
\hline Invierno & 80 & 25,7 \\
\hline Verano & 79 & 25,4 \\
\hline Primavera & 47 & 15,1 \\
\hline \multicolumn{3}{|l|}{ Año $(\mathrm{N}=357)^{*}$} \\
\hline 2014 & 107 & 30 \\
\hline 2015 & 108 & 30,3 \\
\hline 2016 & 63 & 17,6 \\
\hline 2017 & 79 & 22,1 \\
\hline \multicolumn{3}{|c|}{ Procedencia $(\mathrm{N}=307)$} \\
\hline Lima Norte & 204 & 66,4 \\
\hline Lima Centro & 65 & 21,2 \\
\hline Otros $^{2}$ & 38 & 12,4 \\
\hline
\end{tabular}

* Existen 4 pacientes con 2 enfermedades diagnosticadas en diferentes estaciones u años.

${ }^{1}$ Un paciente fue diagnosticado con 2 enfermedades distintas en 2 edades (grupos etarios) distintos.

${ }^{2}$ Otras procedencias como Callao (23), Lima Este (12), Lima Sur (1), Provincia de Barranco (1) y Provincia de Huaral (1). 
Tabla 2. Distribución de las enfermedades infecciosas bacterianas según variables demográficas de los pacientes caninos atendidos en la Clínica Veterinaria Docente Cayetano Heredia en el periodo 2014-2017. Lima - Perú.

\begin{tabular}{|c|c|c|c|c|c|c|c|c|c|c|}
\hline \multirow{3}{*}{ Variable } & \multirow{2}{*}{\multicolumn{2}{|c|}{$\begin{array}{c}\text { Ehrlichiosis } \\
(\mathbf{N}=163)\end{array}$}} & \multirow{2}{*}{\multicolumn{2}{|c|}{$\begin{array}{c}\text { Anaplasmosis } \\
(\mathbf{N}=\mathbf{3 8}) \\
\text { L. canicola } \\
(\mathbf{N}=\mathbf{2 0})\end{array}$}} & \multicolumn{6}{|c|}{ Leptospira } \\
\hline & & & & & \multicolumn{2}{|c|}{$\begin{array}{c}\text { L. } \\
\text { icterohaemo- } \\
\text { rrhagiae } \\
(\mathbf{N}=18)\end{array}$} & \multicolumn{2}{|c|}{$\begin{array}{c}\text { L. } \\
\text { grippotyphosa } \\
(\mathrm{N}=3)\end{array}$} & \multirow[b]{2}{*}{$\mathbf{n}$} & \multirow[b]{2}{*}{$\%$} \\
\hline & $n$ & $\%$ & $n$ & $\%$ & $n$ & $\%$ & $n$ & $\%$ & & \\
\hline \multicolumn{11}{|l|}{ Raza } \\
\hline Mestizo & 71 & 43,5 & 20 & 52,6 & 8 & 40 & 7 & 38,9 & 1 & 33,3 \\
\hline Definida & 92 & 56,5 & 18 & 47,4 & 13 & 60 & 11 & 61,1 & 2 & 66,7 \\
\hline \multicolumn{11}{|l|}{ Sexo } \\
\hline Macho & 105 & 64,4 & 19 & 50 & 15 & 75 & 13 & 72,2 & 3 & 100 \\
\hline Hembra & 58 & 35,6 & 19 & 50 & 5 & 25 & 5 & 27,8 & 0 & 0,0 \\
\hline \multicolumn{11}{|l|}{ Estrato etario } \\
\hline Adulto & 88 & 54 & 24 & 63,2 & 10 & 50 & 8 & 44,4 & 2 & 66,7 \\
\hline Adulto Mayor & 54 & 33,1 & 7 & 18,4 & 7 & 35 & 7 & 38,9 & 1 & 33,3 \\
\hline Cachorro & 21 & 12,9 & 7 & 18,4 & 3 & 15 & 3 & 16,7 & 0 & 0.0 \\
\hline \multicolumn{11}{|l|}{ Estación } \\
\hline Otoño & 56 & 34,4 & 15 & 39,5 & 8 & 40 & 7 & 38,9 & 1 & 33,3 \\
\hline Verano & 44 & 27 & 6 & 15,8 & 3 & 15 & 3 & 16,7 & 1 & 33,3 \\
\hline Invierno & 40 & 24,5 & 13 & 34,2 & 4 & 20 & 5 & 27,8 & 0 & 0,0 \\
\hline Primavera & 23 & 14,1 & 4 & 10,5 & 5 & 25 & 3 & 16,7 & 1 & 33,3 \\
\hline \multicolumn{11}{|l|}{ Año } \\
\hline 2014 & 56 & 34,4 & 8 & 21,1 & 4 & 20 & 2 & 11,1 & 1 & 33,3 \\
\hline 2015 & 53 & 32,5 & 7 & 18,4 & 2 & 10 & 2 & 11,1 & 2 & 66,7 \\
\hline 2016 & 24 & 14,7 & 7 & 18,4 & 7 & 35 & 6 & 33,3 & 0 & 0,0 \\
\hline 2017 & 30 & 18,4 & 16 & 42,1 & 7 & 35 & 8 & 44,5 & 0 & 0,0 \\
\hline \multicolumn{11}{|l|}{ Procedencia } \\
\hline Lima Norte & 106 & 65 & 26 & 68,4 & 16 & 80 & 15 & 83,3 & 2 & 66,7 \\
\hline Lima Centro & 30 & 18,4 & 7 & 18,4 & 1 & 5 & 1 & 5,6 & 1 & 33,3 \\
\hline Otros & 27 & 16,6 & 5 & 13,2 & 4 & 10 & 1 & 5,6 & 0 & 0,0 \\
\hline
\end{tabular}

etario y estación) se presenta en la tabla para el caso de las enfermedades bacterianas y en la tabla 3 para el de enfermedades virales e infecciones parasitarias.

Se encontró $11,5 \%$ de presentación de canes diagnosticados con dos o más agentes etiológicos de enfermedad(es) infecciosa(s) en el periodo 2014-2017, siendo las principales: Anaplasma sp. y Ehrlichia sp. (46.3\%) y L. canicola y L. icterohaemorrhagiae (29,3\%), el detalle de los canes diagnosticados con dos o más agentes etiológicos se encuentra en la tabla 4.

\section{DISCUSIÓN}

Al revisar las historias clínicas de canes atendidosenlaCVDCHpertenecientesalperiodo 2014-2017 de un total de 6006, se obtuvieron 307 $(5,1 \%)$ pacientes con enfermedades infecciosas confirmadas mediante pruebas de laboratorio. Este porcentaje es bajo en comparación a otros estudios realizados en Perú, por ejemplo: en 
Tabla 3. Distribución de las enfermedades infecciosas virales e infecciones parasitarias según variables demográficas de los pacientes caninos atendidos en la Clínica Veterinaria Docente Cayetano Heredia en el periodo 2014-2017. Lima - Perú.

\begin{tabular}{|c|c|c|c|c|c|c|c|c|}
\hline \multirow{3}{*}{ Variable } & \multicolumn{4}{|c|}{ Enfermedades virales } & \multicolumn{4}{|c|}{ Infecciones parasitarias } \\
\hline & \multicolumn{2}{|c|}{$\begin{array}{l}\text { Distemper } \\
\quad(\mathbf{N}=\mathbf{2 8})\end{array}$} & \multicolumn{2}{|c|}{$\begin{array}{l}\text { Parvovirosis } \\
(\mathbf{N}=\mathbf{2 8})\end{array}$} & \multicolumn{2}{|c|}{$\begin{array}{c}\text { Giardiasis } \\
\quad(\mathbf{N}=\mathbf{3 1})\end{array}$} & \multicolumn{2}{|c|}{$\begin{array}{l}\text { Coccidiosis } \\
(\mathbf{N}=\mathbf{2 8})\end{array}$} \\
\hline & $\mathbf{n}$ & $\%$ & $\mathbf{n}$ & $\%$ & $\mathbf{n}$ & $\%$ & $\mathbf{n}$ & $\%$ \\
\hline \multicolumn{9}{|l|}{ Raza } \\
\hline Mestizo & 9 & 32,1 & 9 & 32,1 & 23 & 74,2 & 21 & 75 \\
\hline Definida & 19 & 67,9 & 19 & 67,9 & 8 & 25,8 & 7 & 25 \\
\hline \multicolumn{9}{|l|}{ Sexo } \\
\hline Macho & 15 & 53,6 & 18 & 64,3 & 23 & 74,2 & 13 & 46,4 \\
\hline Hembra & 13 & 46,4 & 10 & 35,7 & 8 & 25,8 & 15 & 53,6 \\
\hline \multicolumn{9}{|l|}{ Estrato etario } \\
\hline Adulto & 9 & 32,1 & 0 & 0,0 & 16 & 51,6 & 22 & 78,5 \\
\hline Adulto Mayor & 1 & 3,6 & 0 & 0,0 & 9 & 29 & 5 & 17,9 \\
\hline Cachorro & 18 & 64,3 & 28 & 100 & 6 & 19,4 & 1 & 3,6 \\
\hline \multicolumn{9}{|l|}{ Estación } \\
\hline Otoño & 6 & 21,4 & 8 & 40 & 10 & 32,3 & 8 & 28,6 \\
\hline Verano & 9 & 32,1 & 3 & 15 & 10 & 32,3 & 9 & 32,1 \\
\hline Invierno & 9 & 32,1 & 4 & 20 & 6 & 19,4 & 8 & 28,6 \\
\hline Primavera & 4 & 14,4 & 5 & 25 & 5 & 16 & 3 & 10,7 \\
\hline \multicolumn{9}{|l|}{ Año } \\
\hline 2014 & 6 & 21,4 & 11 & 39,3 & 6 & 19,4 & 13 & 46,4 \\
\hline 2015 & 8 & 28,6 & 10 & 35,7 & 13 & 41,9 & 11 & 39,3 \\
\hline 2016 & 4 & 14,3 & 3 & 10,7 & 9 & 29 & 3 & 10,7 \\
\hline 2017 & 10 & 35,7 & 4 & 14,3 & 3 & 9,7 & 1 & 3,6 \\
\hline \multicolumn{9}{|l|}{ Procedencia } \\
\hline Lima Norte & 16 & 57,1 & 22 & 78,57 & 19 & 61,3 & 17 & 60,7 \\
\hline Lima Centro & 8 & 28,6 & 5 & 17,86 & 10 & 32,3 & 11 & 39,3 \\
\hline Otros & 4 & 14,3 & 1 & 3,57 & 2 & 6,4 & 0 & 0,0 \\
\hline
\end{tabular}

el laboratorio de Parasitología de la Facultad de Veterinaria y Zootecnia de la Universidad Peruana Cayetano Heredia se observó que un $25 \%$ de pacientes fue positivo a algún tipo parasitosis (Serrano-Martínez et al., 2014) y en una clínica de Tarapoto-Perú se obtuvo un $53.3 \%$ de $\mathrm{CPV}$ en canes con gastroenteritis hemorrágica (Mendoza, 2017).

Es importante señalar que los estudios mencionados fueron diseñados para buscar enfermedades específicas en grupos de pacientes seleccionados, razón por lo que los resultados habrían sido más altos. Además del total de historias clínicas revisadas, muchas tuvieron diagnóstico presuntivo y/o diferencial más no definitivo, es decir, pacientes en los que no se pudo confirmar el diagnóstico con pruebas de laboratorio específicas. Ello podría obedecer a diversos motivos como, la poca disponibilidad económica por parte de los dueños, el estado crítico del paciente lo que puede llevar a un desenlace fatal rápidamente o dueños que solicitan la eutanasia de la mascota (Tovar, 2018). 
Tabla 4. Canes diagnosticados con dos o más agentes etiológicos de enfermedad(es) infecciosa(s) atendidos en la Clínica Veterinaria Docente Cayetano Heredia en el periodo 2014-2017. Lima Perú.

\begin{tabular}{lcc}
\hline \multicolumn{1}{c}{ Agentes Diagnosticados } & n & $\mathbf{\%}$ \\
\hline Anaplasma sp. + Ehrlichia sp. & 19 & 46,3 \\
L. canicola + L. icterohaemorrhagiae & 12 & 29,3 \\
Isospora sp. + Parvovirus canino & 2 & 4,9 \\
Giardia sp. + Isospora sp. & 1 & 2,4 \\
Anaplasma sp. + Parvovirus canino & 1 & 2,4 \\
Ehrlichia sp +L. icterohaemorrhagiae & 1 & 2,4 \\
Ehrlichia sp + Giardia sp. & 1 & 2,4 \\
Ehrlichia sp + Isospora sp. & 1 & 2,4 \\
L. icterohaemorrhagiae + L. grippotyphosa & 1 & 2,4 \\
Anaplasma sp. + L. canicola + L. icterohaemorrhagiae & 1 & 2,4 \\
Ehrlichia sp + L. canicola + L. icterohaemorrhagiae & 1 & 2,4 \\
Total & 41 & 100 \\
\hline
\end{tabular}

Existen otros estudios de presentación de enfermedades infecciosas con un enfoque especifico como, por ejemplo: teniendo en cuenta sólo un agente etiológico como Anaplasma sp, donde se obtuvo 55,1\% de prevalencia (Delgado y Montoya, 2018), otro estudio buscó la presentación de la enfermedad teniendo en cuenta su relación con hemogramas y se obtuvo erliquiosis $(54,29 \%)$ y leptospirosis $(20,95 \%)$ como principales enfermedades infecciosas (Sánchez, 2016). Otro estudio determinó la presencia de parásitos gastrointestinales, obteniendo $25 \%$ positivos a algún tipo parasitosis (Serrano-Martínez et al., 2014). En otros países se obtuvieron porcentajes totales mayores, como en Chile donde se obtuvo $14.8 \%$ de seroprevalencia de leptospirosis (considerando exposición del agente) (Silva y Riedemann, 2007) y en Colombia se obtuvo $67,9 \%$ de parasitosis intestinal (Caraballo et al., 2007).

También se determinó la procedencia de los canes infectados, siendo Lima Norte la procedencia de la mayoría de los pacientes, seguido de Lima Centro y otras procedencias. Se debe tomar en cuenta que estos datos no indican que en esas zonas exista mayor o menor cantidad de presentación de enfermedades infecciosas, y se debe considerar que la frecuencia de esta distribución puede estar influenciada porque la CVDCH se encuentra en Lima Norte. En cuanto a las razas y sexo, se encontró que la mayoría de los canes infectados eran machos de raza definida, esto también puede estar influenciado por la procedencia, ya que de acuerdo con Arauco et al. (2014), existen $56,6 \%$ de canes machos en el distrito de San Martin de Porres con lo que se evidenciaría una mayor preferencia de los propietarios por criar canes de este sexo (Arauco et al., 2014). Al evaluar el grupo etario, se observa que la mayoría de los animales infectados eran adultos. Las posibles causas pueden ser la falta de controles sanitarios en este grupo, como refuerzos de vacunación y desparasitación interna y externa. Otro factor de riesgo puede ser la costumbre de los propietarios de dejar salir a sus mascotas a la calle durante varias horas sin supervisión de la persona responsable, lo que permite una mayor exposición a agentes infecciosos (Arauco et al., 2014).

En cuanto a estacionalidad, hay que considerar que Lima, Perú es un departamento de clima subtropical árido con una temperatura 
y humedad que son relativamente homogéneas a lo largo del año, con temperaturas mensuales que varían de $14.6^{\circ}$ a $28.5^{\circ}$ registradas del 20142016 (INEI, 2017). El estudio demostró que en otoño se diagnosticó la mayoría de los casos, esto no necesariamente indica que en otoño más pacientes hayan sido infectados ni que en esa fecha exista más probabilidad de contagio, ya que hay estudios que indican mayor prevalencia de enfermedades en variadas estaciones.

Una revisión realizada en Brasil halló un aumento de casos de distemper en épocas frías como primavera e invierno, ya que el clima frio facilita el mantenimiento y probabilidad de supervivencia del virus (Headley et al., 2012). En Lima, Perú, se encontró una mayor frecuencia de leptospirosis en las estaciones de primavera-verano con respecto a las de otoño-invierno, sugiriendo que altos niveles de humedad y calor en la atmosfera influyen en la presencia de la enfermedad (Serrano-Martínez et al., 2020). Además, cuando el can contrae el agente infeccioso debe existir un periodo de incubación que, dependiendo de la enfermedad, puede variar en tiempo, por ejemplo, en caso de la Ehrlichia sp puede variar de 2 semanas hasta meses (fase subclínica) para presentar signos clínicos (Waner y Harrus, 2000).

Las principales enfermedades encontradas en el estudio fueron erhlichiosis, leptospirosis, anaplasmosis, giardiasis, coccididiosis, distemper y parvovirosis. Estos resultados coinciden con un estudio similar, realizado en la Clínica Veterinaria Docente Cayetano Heredia, en el periodo 2013 donde según diagnóstico presuntivo, se determinó que las principales enfermedades clínicas halladas fueron ehrlichiosis en primer lugar y leptospirosis en segundo, (Sánchez, 2016).

La ehrlichiosis fue la enfermedad infecciosa más frecuente en el estudio y también se presentó la anaplasmosis, estas son enfermedades transmitidas por garrapatas $y$ fueron diagnosticadas mediante pruebas serológicas
(Test rápido para $E$. canis Anigen $A b \AA$ y SNAP 4Dx Plus de IDEXX®). Ambas se observaron en canes adultos mestizos, aunque se sabe que no hay predilección por sexo, edad ni raza para la infección por estos agentes etiológicos (Waner y Harrus, 2000; Delgado y Montoya, 2018). La alta frecuencia de estas enfermedades se justificaría debido a la alta prevalencia de $R$. sanguineus en Lima Norte, vector de estas infecciones (Ferrel, 2014; Estares et al., 2000).

La leptospirosis fue otro hallazgo importante en este estudio. Mediante la prueba de microaglutinación (MAT) se encontraron los serovares L. canicola, L. icterohaemorrhagiae y L. grippotyphosa, resultados similares a los encontrados en un estudio previo en Lima (Siuce et al., 2015). De acuerdo con estos hallazgos, se observa que Lima tiene condiciones epidemiológicas favorables para su sobrevivencia e infecte a los canes y que por su carácter zoonótico se debe advertir de su presencia a los propietarios de los canes a fin de que tomen las medidas preventivas para evitar la exposición humana a la bacteria.

La mayoría de los diagnósticos de Leptospira se realizó en otoño, sin embargo, en otro estudio en Lima se encontró una mayor frecuencia en las estaciones de primavera-verano (SerranoMartinez et al, 2020). Cabe señalar que en los años 2016 y 2017 se dio el fenómeno del Niño Costero lo que puede ser un factor por el cual se observó una cantidad considerable de casos clínicos, ya que se produjo un aumento de humedad por las lluvias intensas y aumento de temperatura favoreciendo la permanencia del agente patógeno en el medio ambiente.

La mayor presentación de coccidiosis se produjo en cachorros, mientras que para giardiasis fue más frecuente en jóvenes y adultos. La presencia de giardiasis representa un peligro para la salud pública al ser zoonótico y en caso de la coccidiosis esta se considera como parásito oportunista que se presenta 
principalmente en animales con cierto grado de inmunosupresión (Vega et al., 2014). Se debe realizar exámenes coproparasitológicos a los pacientes que tengan diarrea ya que es posible que estos agentes patógenos se encuentran involucrados en estos cuadros. En cuanto a la estación de mayor presentación fue verano para ambas enfermedades lo que condiciona un clima apropiado para el desarrollo de estos protozoarios (European Scientific Counsel Companion Animal Parasites, 2013), lo que no descarta su presentación en otras estaciones del año.

Los pacientes con diagnóstico de distemper y parvovirosis, ambos confirmado mediante pruebas de inmunocromatografía (Anigen ${ }^{\circledR}$ Rapid CDV test kit y test rápido Anigen para CPV Ag), fueron en su mayoría cachorros, probablemente porque presentan mayor susceptibilidad a infecciones debido a la disminución de la inmunidad maternal, falta de vacunación, y estado de desnutrición (Duijvestijn et al., 2016; Duque et al., 2017; Headley et al., 2012). Cabe señalar, que algunas mascotas sí contaban con registros de vacunación actualizados y aun así presentaron la enfermedad. Ello puede estar asociado a la presencia de una diversidad genética del agente patógeno, un mal procedimiento de la vacunación o interferencia de la vacunación con altos títulos de anticuerpos maternos (Morales, 2016).

En el estudio se evidenció pacientes que tienen más de un diagnóstico confirmatorio. Anaplasma sp. y Ehrlichia sp, fueron encontrados simultáneamente, tal cual se han observado en otros estudios donde se observaron coinfecciones para estas infecciones que tiene un vector común, para el caso la garrapata parda de los canes (Dolz et al., 2013; McCown et al., 2013). El diagnóstico de estas infecciones parasitarias fue realizada mediante prueba rápida de inmunoensayo enzimático o ELISA
(SNAP 4Dx Plus de IDEXX®). Asimismo, se encontró pacientes con presencia de dos serovariedades de leptospira (L. canicola y $L$. icterohaemorrhagiae). Estudios reportan casos de canes que pudieron pasar por una infección a determinado serovar y luego se infectaron con otro serovar de distinto serogrupo (Silva y Riedemann, 2007; Siuce, 2015). Así mismo, también existen reacciones cruzadas entre serovares y se explican por presencia de antígenos compartidos entre serovares y grupos (Rodríguez et al., 2004).

\section{CONCLUSIONES}

La frecuencia de enfermedades infecciosas confirmadas mediante pruebas de laboratorio específicas en canes en la CVDCH fue de 5,1\% (307/6006) durante el periodo 2014-2017. Las enfermedades infecciosas predominantes encontradas fueron erliquiosis $(45,5 \%) \mathrm{y}$ leptospirosis (11,5\%) Además, en el estudio se evidencio la posibilidad de detectar canes infectados con dos o más agentes etiológicos infecciosos, correspondiendo a un $11.5 \%$, siendo la principal infección mixta causada por Erlichia sp. y Anaplasma sp.

El estudio provee información acerca de las enfermedades infecciosas que con mayor frecuencia se presentan en clientes que solicitan los servicios de la CVDCH. Ello permite tener una aproximación al comportamiento de la demanda de diagnósticos y tratamientos, así comopreveniry controlarposibles enfermedades en poblaciones de canes de la zona; así como orientar al veterinario clínico en el diagnóstico de las enfermedades transmisibles de mayor presentación, favoreciendo un mejor manejo terapéutico y/o profiláctico.

\section{Correspondencia:}

Erika Zuñiga Mendizabal

Correo electrónico:erika.zuniga.m@upch.pe 


\section{REFERENCIAS BIBLIOGRAFICAS}

1. Acosta-Jamett, G., Surot, D., Cortés, M., Marambio, V., Valenzuela, C., Vallverdu, A., \& Ward, M.P. (2015). Epidemiology of canine distemper and canine parvovirus in domestic dogs in urban and rural areas of the AraucaníaregioninChile. Vetmicrobiology, 178(3), 260-264.

2. Arauco, D., Urbina, B., León, D., \& Falcón, N. (2014). Indicadores Demográficos y Estimación de la Población de canes con dueño en el distrito de San Martin de Porres, Lima-Perú. Salud tecnol vet, 2(2), 83-92.

3. Ballweber, L.R., Xiao, L., Bowman, D.D., Kahn, G., \& Cama, V.A. 2010. Giardiasis in dogs and cats: update on epidemiology and public health significance. Trends in parasitology, 26(4), 180-189.

4. Bartges, J., Boynton, B., Hoyumpa-Vogt, A., Krauter, E., Lambrecht, K., Svec, R., \& Thompson, S. (2012). AAHA Canine Life Stage Guidelines. Journal of the American Animal Hospital Association, 48 (1), 4.

5. Caraballo, A., Jaramillo, A., \& Loaiza, J. (2007). Prevalencia de parásitos intestinales en caninos atendidos en el Centro de Veterinaria y Zootecnia de la Universidad CES, 2007. Revista CES Medicina Veterinaria y Zootecnia, 2 (2), 24-31.

6. Couto, G. (2005). Medicina interna de animales pequeños. Intermédica.

7. Cusicanqui, J. (2018). Frecuencia serológica de Ehrlichia canis en pacientes caninos sospechosos a la enfermedad durante el periodo 2014-2016 en Lima Norte. [Tesis de Médico Veterinario Zootecnista]. Universidad Peruana Cayetano Heredia.

8. Delgado, N., \& Montoya, A.M. (2018). Influencia de la edad y el sexo sobre la prevalencia de Anaplasma Spp en caninos (canis familiaris) atendidos en Clínicas Veterinarias en los Distritos de José Leonardo Ortiz, La Victoria y Chiclayo. Julio-Diciembre 2017. [Tesis de Médico Veterinario]. Universidad Nacional Pedro Ruiz Gallo.

9. Dolz, G., Ábrego, L., Romero, L.E., CamposCalderón, L., Bouza-Mora, L., \& Jiménez-Rocha, A.E. (2013) . Ehrlichiosis and anaplasmosis in Costa Rica. Acta Médica Costarricense, 55, 34-40.

10. Duijvestijn, M., Mughini-Gras, L., Schuurman, N., Schijf, W., Wagenaar, J.A., \& Egberink, H. (2016). Enteropathogen infections in canine puppies : (Co-) occurrence, clinical relevance and risk factors. Vet Microbiology, 195, 115-122.

11. Duque-García, Y., Echeverri-Zuluaga, M., TrejosSuarez, J., \& Ruiz-Saenz, J. (2017). Prevalence and molecular epidemiology of Canine parvovirus 2 in diarrheic dogs in Colombia, South America: A possible new CPV-2a is emerging? Vet Microbiology, 201, 56-61.
12. European Scientific Counsel Companion Animal Parasites. (2013). Control de Protozoos Intestinales en Perros y Gatos. European Scientific Counsel Companion Animal Parasites

13. Estares, L., Chávez, A., \& Casas, E. (2000). Ectoparásitos en caninos de los distritos de la zona climática norte de Lima Metropolitana. Rev Invest Vet Perú, 11, 72-76.

14. Fascetti, A.J., \& Delaney, S.J. (2012). Applied Veterinary Clinical Nutrition. Wiley Blackwell.

15. Federation Cynologique Internationale. (2016). Nomenclatura de las razas de la FCI. Federation Cynologique Internationale. http://www.fci.be/es/ Nomenclature/Ferrel, E. (2014). Identificación de ectoparásitos en canis familiaris de distritos del cono este de Lima Metropolitana. [Tesis de Médico Veterinario Zootecnista]. Universidad Peruana Cayetano Heredia.

16. Greene, C. (2000). Enfermedades infecciosas en perros y gatos. MgGraw-Hill Interamericana.

17. Headley, S.A., Amude, A.M., Alfieri, A.F., Bracarense, A.P.F., \& Alfieri, A.A. (2012). Epidemiological features and the neuropathological manifestations of canine distemper virus-induced infections in Brazil: a review. Semina: Ciências Agrárias, 33(5), 1958.

18. Instituto Nacional de Estadística e Informática. (2014). Una Mirada a Lima Metropolitana. Instituto Nacional de Estadística e Informática. https://www. inei.gob.pe/media/MenuRecursivo/publicaciones digitales/Est/Lib1168/libro.pdf

19. Instituto Nacional de Estadística e Informática. (2017). Perú Anuario de Estadísticas Ambientales 2017. Instituto Nacional de Estadística e Informática. https://www.inei.gob.pe/media/MenuRecursivo/ publicaciones_digitales/Est/Lib1469/libro.pdf

20. Llanos, M., Condori, M., Ibáñez, T., \& LozaMurguia, M. (2010). Parasitósis entérica en caninos (Canis familiaris) en el área urbana de Coroico, Nor Yungas Departamento de La Paz, Bolivia. Journal of the Selva Andina Research Society, 1 (1), 37-49.

21. López, J., Abarca, K., Paredes, P., \& Inzunza, E. (2006). Parásitos intestinales en caninos y felinos con cuadros digestivos en Santiago, Chile: Consideraciones en Salud Pública. Revista médica de Chile, 134(2), 193-200.

22. Luna, A.M.A., Moles, C.L.P., Gavaldón, R.D., Nava, V.C., \& Salazar, G.F. (2008). La leptospirosis canina y su problemática en México. Revista de salud animal, 30(1), 01-11.

23. McCown, M.E., Monterroso, V.H., \& Cardona, W. (2015). Surveillance for Ehrlichia canis, Anaplasma phagocytophilum, Borreliaburgdorferi, and Dirofilaria immitis in Dogs From Three Cities in Colombia. CES Medicina Veterinaria y Zootecnia, 10(2), 224-231.

24. Mendoza, C. (2017). Diagnóstico de Parvovirus canino mediante el método del Rapid kit Cpv Ag en 
pacientes con gastroenteritis hemorrágica en el distrito de Tarapoto. [Tesis de Médico Veterinario]. Universidad Nacional de San Martín.

25. Monteiro, F.L., Cargnelutti, J.F., Martins, M., Anziliero, D., Erhardt, M.M., Weiblen, R., \& Flores, E.F. (2016). Detection of respiratory viruses in shelter dogs maintained under varying environmental conditions. Brazilian Journal Of Microbiology, 47(4), 876-881.

26. Morales, D. (2016). Determinación de anticuerpos IgG contra parvovirus canino tipo 2 en perros inmunizados con dos protocolos de vacunación. [Tesis de Médico Veterinario Zootecnista]. Universidad Autónoma del Estado de México.

27. Kumar, V., Abbas, A., Fausto, N., \& Aster, J. (2010). Robbins y Cotran. Patología estructural y funcional. Elsevier.

28. Rodríguez, A.L., Ferro, B.E., Varona, M.X., \& Santafé, M. (2004). Evidencia de exposición a Leptospira en perros callejeros de Cali. Biomédica, 24(3), 291-5.

29. Sánchez, A.D.R. (2016). Frecuencia y asociación de alteraciones hematológicas según diagnóstico presuntivo en pacientes caninos atendidos en la Clínica Veterinaria Cayetano Heredia en el periodo 2013. [Tesis de grado Médico Veterinario]. Universidad Peruana Cayetano Heredia.

30. Schudel, A.A., \& van Gelderen, C. (2002). Enfermedades infecciosas de los animales. Revista CienciaHoy en línea, 11(66). http://www.cienciahoy. org.ar/ch/ln/hoy66/enfermedades.htm

31. Serrano-Martínez, E., Tantaleán, M., Castro, V., Quispe, M., \& Casas, G. (2014). Retrospective study of parasite frequency in faecal samples in routine laboratory analysis. Rev investig vet Perú, 25(1), 113-116.

32. Serrano-Martínez, E., Burga, C., Hinostroza, M.E., \& Zúñiga, F. R. (2020). Influencia de las estaciones climáticas en la presencia de leptospirosis canina en el norte y centro de Lima, Perú. Revista de Investigaciones Veterinarias del Perú, 31(4), e19018. https://doi.org/10.15381/rivep.v31i4.19018
33. Silva, R.F., \& Riedemann, S. (2007). Seroprevalencia de leptospirosis canina en perros atendidos en clínicas veterinarias, mediante aglutinación microscópica y comparación con las técnicas de aislamiento e inmunofluorescencia indirecta. Archivos de medicina veterinaria, 39(3), 269-274.

34. Siuce, J., Calle, S., Pinto, C., Pacheco, G., \& Salvatierra, G. (2015). Identificación de Serogrupos Patógenos de Leptospira en Canes Domésticos. Rev investig vet Perú, 26(4), 664-675.

35. Sosa-Gutierrez, C., Quintero, M., Gaxiola, S., Cota, S., Esteve-Gassent, M., \& Gordillo-Pérez, M. (2013). Frequency and clinical epidemiology of canine monocytic ehrlichiosis in dogs infested with ticks from Sinaloa, Mexico. Journal of veterinary medicine, 2013 (797019), 1-3.

36. Sotelo, P., Chávez, V., Casas, A., Pinedo, R., \& Falcón, N. (2013). Giardiasis y criptosporidiasis en caninos de los distritos del Cono Oeste de Lima Metropolitana. Rev investig vet Perú, 24(3), 353-359.

37. Soto, R. (2017). Detección molecular del virus del distemper canino en casos clínicos de caninos domésticos no vacunados y determinación de los factores de riesgo. [Tesis de Médico Veterinario] Universidad Nacional de Mayor de San Marcos

38. Tateishi, V., Lí, O., Hoyos, L., Rivera, H., Manchego, A., Barrios, L., \& More, J. (2015). Identificación hematológica y molecular de Anaplasma platysen en caninos domésticos de Lima Metropolitana con signos clínicos compatibles con anaplasmosis. Rev investig. vet Perú, 26(1), 111-118.

39. Tovar MG. Caracterización de la casuística de caninos atendidos en el servicio de cuidados intermedios de la CVCH en el periodo 2014 - 2016. [Tesis de Médico Veterinario Zootecnista]. Universidad Peruana Cayetano Heredia.

40. Veja, S., Serrano-Martínez, E., Grandez, R., Pilco, M., \& Quispe, M. (2014). Parásitos gastrointestinales en cachorros caninos provenientes de la venta comercial en el Cercado de Lima. Salud Tecnol Vet, 2 , 71-77.

41. Waner, T., \& Harrus, S. (2000) . Ehrlichiosis monocitica canina. International Veterinary Information Service. 